\title{
HBASE Performance Analysis in Big Datasets Processing
}

\author{
Tsvetelina Mladenova, Yordan Kalmukov, Milko Marinov, Irena Valova \\ Department of Computer Systems and Technologies, University of Ruse, 8 Studentska Str., \\ 7017 Ruse Bulgaria
}

\begin{abstract}
The term Big Data has gained popularity in recent years due to technological developments and the accumulation of data from various sources, mobile devices and sensors. Hbase is a distributed open source environment that uses available disk space optimally and efficiently based on data. It organizes data in a very different way from standard relational databases and works with both structured and unstructured data. This article describes our experience and research on how the execution time for inserting datasets and selecting data depends on the size of the data volumes, the locations (nodes of the same or different networks) from which they send or retrieve and what is the effect of the selected data organization (especially RowKey design) on the execution time.
\end{abstract}

Keywords - Big Data, No SQL, HBase, Thrift server, insert, select

\section{Introduction and Related Work}

The term Big Data is being used to describe a big volume of structured and non-structured data that is big enough and can't be processed by traditional methods for database management.

DOI: 10.18421/TEM103-07

https://doi.org/10.18421/TEM103-07

Corresponding author: Irena Valova,

Department of Computer Systems and Technologies,

University of Ruse, 8 Studentska Str., 7017 Ruse, Bulgaria.

Email: ivalova@ecs.uni-ruse.bg

Received: 27 March 2021.

Revised: 26 June 2021.

Accepted: 03 July 2021.

Published: 27 August 2021.

(c) BY-NC-ND (C) 2021 Tsvetelina Mladenova et al; published by UIKTEN. This work is licensed under the Creative Commons Attribution-NonCommercial-NoDerivs 4.0 License.

The article is published with Open Access at www.temjournal.com
In most cases, the data is dynamic and changes too fast, making it unsuitable for processing by existing applications but has the potential to help make faster and intelligent business decisions. In order for this potential to be achieved, appropriate technological infrastructure is needed.

Doug Laney [1] describes and defines Big Data with the definition of the three Vs: "Big Data as a circumstance where the volume, velocity and variety of data of an organization's storage go beyond the computation capacity for precise and well-timed decision making". Lee [2] also uses the three Vs: Volume, Velocity and Variety. Other researchers propose four Vs as they add Veracity as a characteristic of Big Data [4], [5]. In addition to the four Vs, [6] proposes a fifth V - Value. Figure 1. shows the five main characteristics when defining Big Data. Big Data is not something new. In practice people have been trying to plan and make decision on the base of gathered data. The term has been gaining popularity in recent years because of the technological development and the accumulation of data from various sources. According to [3], the evolution of the big data is divided to three phases: structured data that is stored and managed by DBMS; web-base, non-structured data; data that comes from mobile devices and sensors.

In order for the big data to be useful, some tools for storage, processing and analysing are needed. The real value of the big data is the possibility to analyse the trends in the data in real-time. That way some critical data can be derived. It turns out that the standard relational databases are not suitable and new ways should be developed and used. This kind of tools can be NoSQL databases. [8] makes a comparison between RDBMS and NoSQL. NoSQL can be interpreted as non-relational or not SQL databases or not only SQL databases. At least for now, the second interpretation is accepted, as the management tools for NoSQL databases usually not only manages the non-relational databases but also supports some kind of SQL-like language for working with data. 


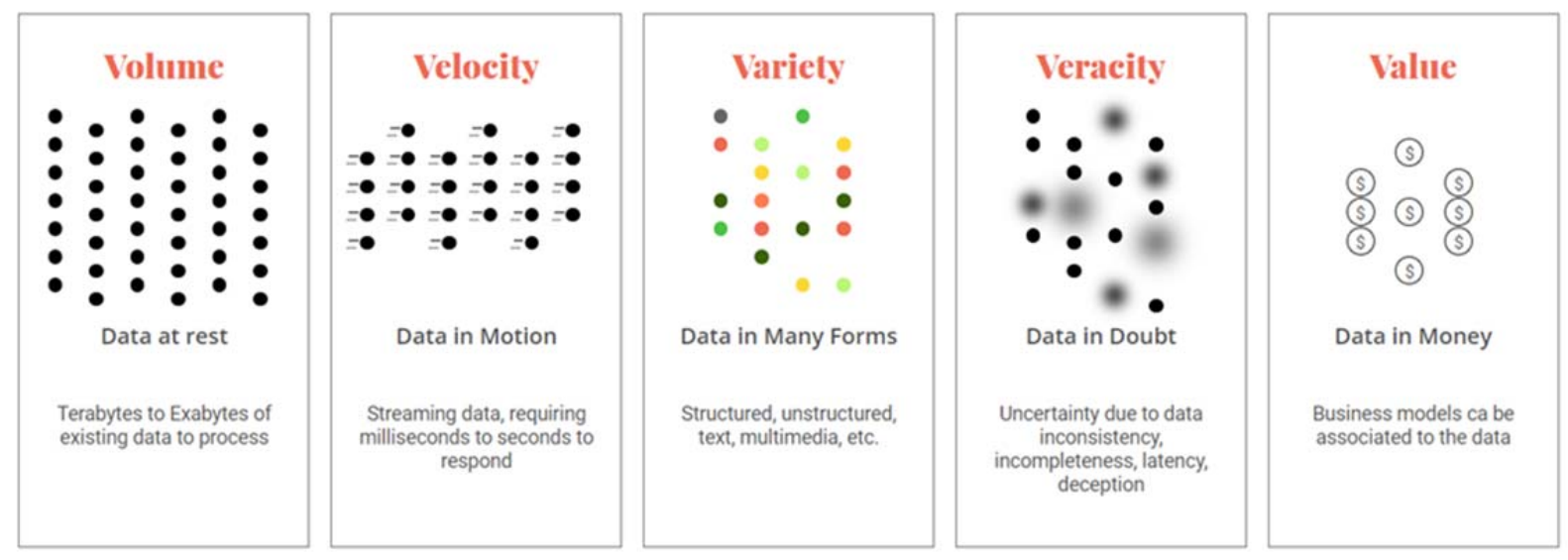

Figure 1. Five main characteristics for the Big Data definition

Hbase [11] is one of the environments that works with Big Data. It is open-source distributed environment that uses the available disk space optimally and effectively depending on the data. This system organizes the data in a very different way to the standard relational databases [8], [9], [10] and works with both structured and non-structured data. HBase is made of three main components - Hmaster, HRegion Server and HBaseClient + Zookeeper [7]. It is built to work in a cluster computer system where each node has got its own role. HBase divides the tables into regions which are hosted by servers called Region Servers. This is a column-based database and the tables within it are saved by row. Column Families are defined by pairs of type KEY - value and are collections of columns. Each table contains several column families and each of them can have any number of columns. Successional values of columns are stored in order and continuously on the disk. The smallest unit is a cell. The format of storing a piece of data in a cell is as follows: tuple (row, column, version). Each cell value from the table has a timestamp or a version that shows the chronological order of cell updates.

Typically, the cell in HBase contains the following components:

- RowKey,

- Column Family,

- Column Qualifier,

- Column Value,

- Timestamp.

The last two determine the cell version and if a timestamp was not assigned when the data was saved, an automatic timestamp is assigned based on the system time of server region where the region is currently based. A cell may have different values in different timestamps. While RDBMS uses a database scheme which defines the structure of the tables and the connections between them, HBase does not have a scheme and the tables do not have a fixed number of columns, the only thing defined is the column families and it is not necessary to aim to normalize the data in them. Hbase is suitable for wide tables and is horizontally scalable. The tables in HBase are partitioned based on interval values from the key. Row Key is used to search for entries similar to keys in RDBMS.

The main goal of this experimental research is to see how the two main operations - inserting new datasests and selecting or retrieving data work in databases with HBase as an environment that works with big data and supports not relational databases. Also, with this experiment we try to compare how the RowKey design affects the execution time of these two operations - the Hbase-recommended RowKey design and the more "relational-like" RowKey design have been used in the experiments.

\section{Dataset Description}

The whole data set consist of over 490000000 entries from various measurement sensors. The number of entries is enough so that we can say the dataset covers the Volume factor of the definition of Big Data and thus can be classified as such. The data entries have a structure shown in Table 1. and are gathered from 250 households across the UK and Bulgaria. The whole dataset can be classified as mostly numerical.

\section{Experiment Methodology}

The conducted experiment has been performed on the hardware, described in Figure 2. Every test case has been run three times and the average result from every test scenario has been taken into consideration. The purpose of the three times test running is to rule out the possibility of atypical behavior and thus give results that are more accurate. 
Table 1. The structure of the data entries

\begin{tabular}{|c|c|c|}
\hline $\begin{array}{c}\text { Dataset } \\
\text { Name }\end{array}$ & $\begin{array}{c}\text { Column } \\
\text { Family Name }\end{array}$ & Description \\
\hline house_id & house:mac & $\begin{array}{c}\text { MAC address of the } \\
\text { house }\end{array}$ \\
\hline date_time & house:datetime & $\begin{array}{c}\text { Timestamp of the } \\
\text { measurement }\end{array}$ \\
\hline ch1_watts & ch:1 & ch:2 \\
\hline ch2_watts & ch:3 & $\begin{array}{c}\text { The temperature in } \\
\text { the building }\end{array}$ \\
\hline ch3_watts & readings:temp \\
temp & consumption \\
gas_reading & readings:gas & $\begin{array}{c}\text { indicator at the time } \\
\text { of the measurement }\end{array}$ \\
\hline $\begin{array}{c}\text { appliance_1 } \\
\text { to } \\
\text { appliance_10 }\end{array}$ & app:1 & $\begin{array}{c}\text { The measured } \\
\text { indicator from the } \\
\text { first appliance }\end{array}$ \\
\hline
\end{tabular}

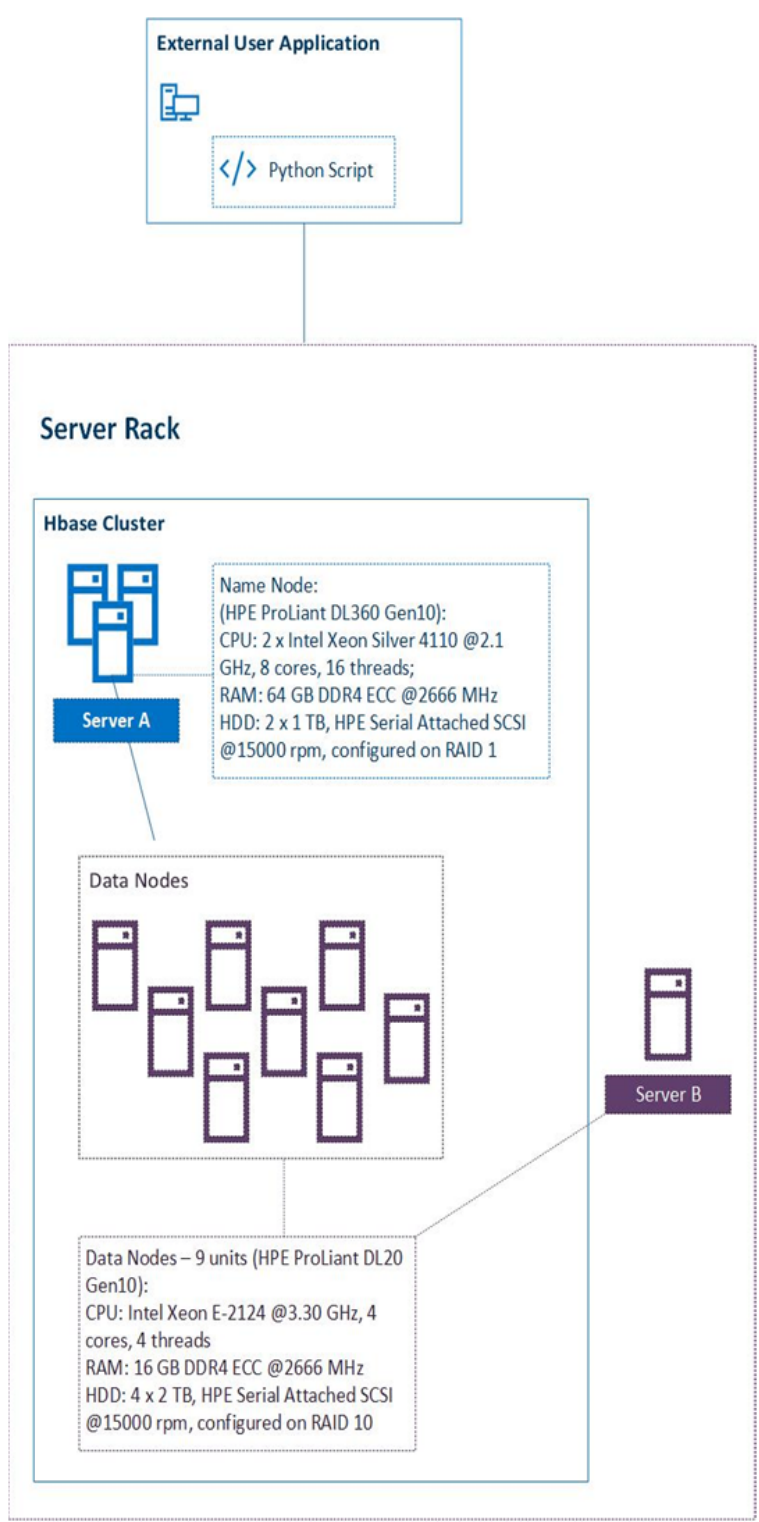

Figure 2. Hardware architecture for the experiment
Every test case is in the form of a Python script that is loaded on the server along with the data. Every test scenario uses the happybase python library for interactions with Apache Hbase. The library is based on the ThiftPy2 library to connect to the Hbase Thrift Server. With easy to understand methods and documentation, the library is a preferred way to connect to Hbase via Python for many developers and real-life situations.

The versions used in the experiment are described in Table 2.

Table 2. Software versions used in the experiment

\begin{tabular}{|c|c|c|}
\hline TYPE & Server A & Server B \\
\hline Python & 2.7 .5 & 3.6 .8 \\
\hline HappyBase & 1.2 .0 & 1.2 .0 \\
\hline ThriftPy2 & 0.4 .12 & 0.4 .12 \\
\hline
\end{tabular}

\section{Test Cases}

For the purposes of this experiment, a total of two test scenarios were conducted - test case INSERT and test case SELECT. Every test scenario consists of subtests as shown in Table 3. and Table 4.

The differences between the subtests are:

- the sources of the data, which is being send to the Thrift Server,

- the type of the RowKeys Design,

- the data size, depending on the RowKey design.

Server A, the Name Node of the cluster, performs test scenarios INSERT.3, INSERT.3.1, INSERT.4, SELECT.2, SELECT.4, SELECT.4.1 and SELECT.4.2.

Server B, which is located in the same rack as the Hbase cluster, but is not part of it, and thus can be defined as an external server, performs test scenarios INSERT.1, INSERT.1.1, INSERT.2, INSERT.2.1 and SELECT.1, SELECT.3, SELECT.3.1 and SELECT.3.2.

When designing the table schema and more specifically, the RowKeys, it should be noted that there are several guidelines to be followed. It also should be noted that the distribution of the data across the nodes is based on the RowKeys - every Hbase region is storing the data between "start RowKey" and "end RowKey". Meaning that all of the cells that have similar keys are placed in one region. Wrong design of the key can lead to the socalled "hot spotting" - placing all of the data in one or few regions instead of distributing it across the data nodes and concentrating the read/write operations to few regions of the table. 
Table 3. Test scenarios for INSERT operations

\begin{tabular}{|c|c|c|c|}
\hline Test Scenario & Insert From & RowKeys Type & Dataset \\
\hline INSERT.1 & External Server & Relational & A \\
\hline INSERT.1.1 & External Server & Relational & B \\
\hline INSERT.2 & External Server & Non-Relational & B \\
\hline INSERT.2.1 & External Server & Salted Non-Relational & A \\
\hline INSERT.3 & Name Node & Relational & A \\
\hline INSERT.3.1 & Name Node & Non-Relational & B \\
\hline INSERT.4 & Name Node & B \\
\hline
\end{tabular}

Table 4. Test scenarios for SELECT operations

\begin{tabular}{|c|c|c|c|c|}
\hline Test Scenario & Insert From & RowKeys Type & Dataset & Select Clause \\
\hline SELECT.1 & External Server & Relational & A & 1 row \\
\hline SELECT.2 & Name Node & Relational & A & 1 row \\
\hline SELECT.3 & External Node & Relational & A & $25 \%$ of the rows \\
\hline SELECT.3.1 & External Node & Relational & A & $50 \%$ of the rows \\
\hline SELECT.3.2 & External Node & Relational & A & $75 \%$ of the rows \\
\hline SELECT.4 & Name Node & Relational & A & $25 \%$ of the rows \\
\hline SELECT.4.1 & Name Node & Relational & A & $50 \%$ of the rows \\
\hline SELECT.4.2 & Name Node & Relational & A & $75 \%$ of the rows \\
\hline
\end{tabular}

When designing the RowKey, the following steps should be done:

- Ensuring that a unique RowKey is selected or constructed if none is present;

- Preventing the "hot spotting" with the help of hashing or salting of the RowKey.

The current experiment contains a test case with a salted RowKey (INSERT.2.1) where the relationallike key from Dataset $\mathrm{A}$ is prefixed with a random letter from the English alphabet. That ensures the placement of the data in several regions and thus preventing the "hot spotting".

The relational type of RowKeys consist of the mac address of the house concatenated with the timestamp of the measurement, while the nonrelational type consists only of the mac address. Every measurement is saved as a version of the row corresponding to the mac address.

Characteristically for Hbase is the support of the versions. In relational databases when we try to insert two data rows with the same key, the database will return an error message, stating that the row cannot be inserted because of duplication. This is not the case with Hbase - where we can insert multiple rows with same RowKeys and every Nth row will be inserted as a new version of the row. The default value of the versions is three and can be increased as needed when creating the database. When creating the database through the Thrift API the variable is called max_versions.

Because of the nature of our data, two sub-datasets were used - dataset A and dataset B. Dataset A is used when we tested the relational type of the RowKeys and dataset B is used when we tested the non-relational type of the RowKeys. A more in-depth description of the datasets and the database schema can be seen in Table 5. When executing the test scenarios with the relational RowKey we can have as much as $490 \mathrm{~m}$ unique rows, as the RowKey is a combination of the mac address and the timestamp. But when we want to test the Non-Relational form of the RowKeys we should leave the timestamp and use only the mac address, which in turn leaves us with 237 unique rows (as this is the number of the unique mac addresses). In order to achieve similar data sizes for both test scenarios, when executing test cases with dataset $\mathrm{B}$ we use an increasing number of the versions in which we store the data from the unique timestamp measurements.

It's worth noting that while performing the experiments, server $\mathrm{B}$ is loaded only with the execution of the experiment and server A (the Name Node) is loaded with the performing of the experiment and the distribution of the Cloudera Manager tasks and services.

Table 5. Description of the datasets and the database schema

\begin{tabular}{|c|c|c|c|}
\hline Dataset & Workloads & $\begin{array}{c}\text { Unique } \\
\text { RowKeys }\end{array}$ & Versions \\
\hline & 200000 & 200000 & \\
A & 400000 & 400000 & \\
& 600000 & 600000 & 3 \\
& 800000 & 800000 & \\
\hline & 1000000 & 1000000 & \\
\hline & 184255 & & 800 \\
B & 358443 & & 1600 \\
& 518751 & 237 & 2400 \\
& 678246 & & 3200 \\
& 837446 & & 4000 \\
\hline
\end{tabular}




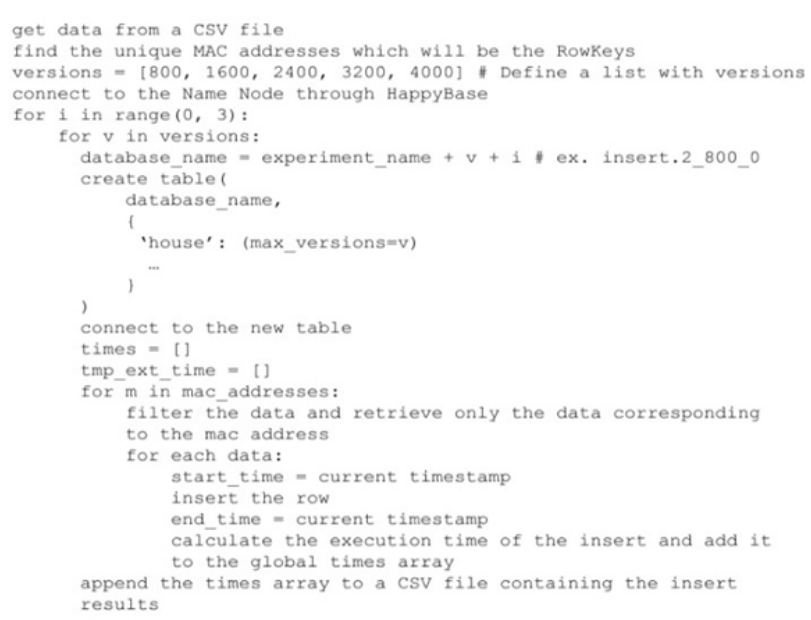

Figure 3. The example piece of code for test case INSERT.2

\section{Python Code}

The example piece of code in Python for test case INSERT.2 can be seen in Figure 3. The other test cases are conducted in a similar manner.

\section{Results with Insert}

The average number of requests per second when inserting multiple records varies between 360 and 420. The execution of multiple insert queries is generally faster when it's coming from Server B, the external server, as seen in Figure 4., and slower when the data is coming from the Name Node, located in the same cluster. That can be due to the workload of the Name Node and the constant execution of additional tasks coming from the Cloudera Manager.

The same figure shows that when performing insert operation, the design of the RowKey has little to no effect on the execution time. The initiator of the insert queries, the source of the experiment, had more effect on the execution than the design of the RowKey.

Furthermore, the number of the versions and the manner of the insert, as a new version or as a new row altogether, has no effect on the execution time.

The same operation, but with dataset A and only from the External Server, can be seen in Figure 5. The figure shows that the execution time does not change in noticeable manner even when the inserted rows does not have any versions (the brown line) and are salted (the red line).

Concluding the experiments in the INSERT series, we can say that the number of the requests that are being processed per second does not change regardless of the RowKey design nor the salting techniques. What causes a difference is the source of the requests - the ones coming from a server inside the server's rack, but not in the cluster, are being processed faster than the ones coming from the Name Node.

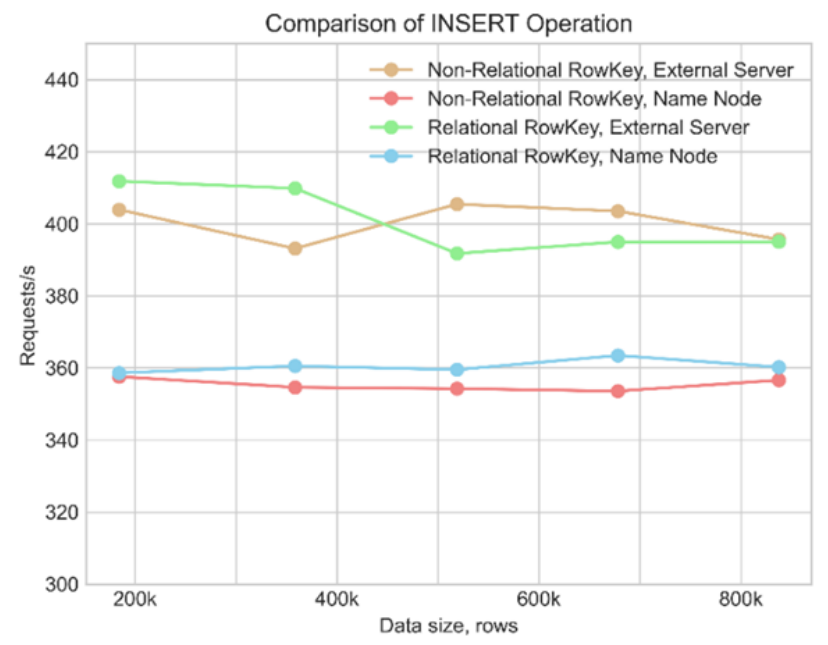

Figure 4. Comparison of Insert operation with different RowKey Designs and Data Sources, dataset B (INSERT.1.1, INSERT.1.4, INSERT.1.2, INSERT.4)

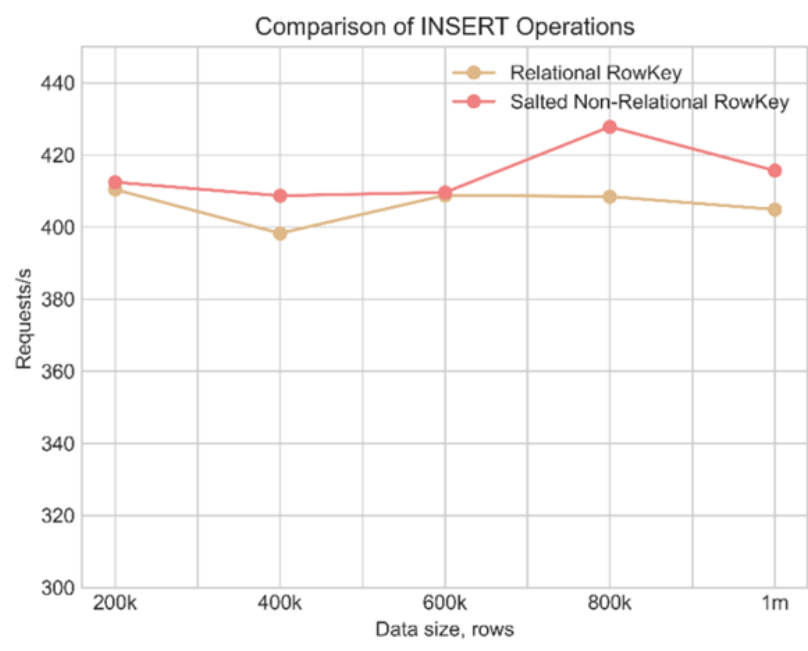

Figure 5. Comparison of Insert operation with different RowKeys Designs, dataset A

\section{Results with Select}

The SELECT test cases were conducted only on dataset A. The decision to test only the first dataset comes from the volume of the data and the limitations of the Thrift API that is used to query the data from Hbase. When selecting data from Hbase with the help of the Thrift API only the last version of the row is returned. For example, if we have a row with 4000 versions, as we have in the test cases with dataset B, we can retrieve only the last inserted item. Moreover, since we have only 237 unique rows, we have decided that every test case with only 237 rows should be considered insignificant, as it does not represent the characteristics of Big Data. Therefore, all of the SELECT experiments are done on dataset A. 
Figure 6. displays the difference when selecting one random row from the whole dataset. The experiments are conducted on databases with different sizes and from the different sources - the External Server and the Name Node. Every select query is performed three times and every time the selected row is chosen randomly so that caching of the query is avoided. As seen in the figure, the execution time varies between 40 and 60 milliseconds and there is essentially no difference between the two servers.

As seen in Figure 7. the execution time of the SELECT operation increases rectilinearly with the increase of the queried rows. And while the Name Node performed slightly worse than the External Server in the INSERT experiments, the SELECT test cases shows that it is quite faster when performing the SELECT test experiment. Comparing two select tests from the External Server, one with relational RowKey design and one with salted non-relational design, it should be noted that the salted design performs better than the other does. The figure combines test SELECT.3 to SELECT.4.2.

\section{Conclusions}

This paper examines the execution time of the two main operations used when managing data. A series of experiments are held and analysed. Two sets of data have been tested, from two different data sources. The results from the experiments have been compared and considered:

- The execution of the Insert queries is generally faster, coming from the External server and the design of the RowKey does not affect the number of the requests that are being processed per second.

- The number of the cells versions is also not a key factor in the execution time. There is no difference between insertion of data with a number of versions equal to three and insertion of data with a number of versions equal to 4000 .

- The time of the execution of a select query is almost the same when selecting one random row from the table regardless of the source that is initiating the selection.
- In contrast to the results obtained from the Insert tests, the Select tests show that the Name Node is performing better. When the source of the select query is the Name Node, the results are sent back faster to the client.

Areas for future improvement have been identified. Some of the future work will consist of:

- Testing the role of the compression when performing the Insert and Select operations in Hbase;

- Testing the Insert and Select operations from multiple devices, thus simulating the parallel work of several different data sources.

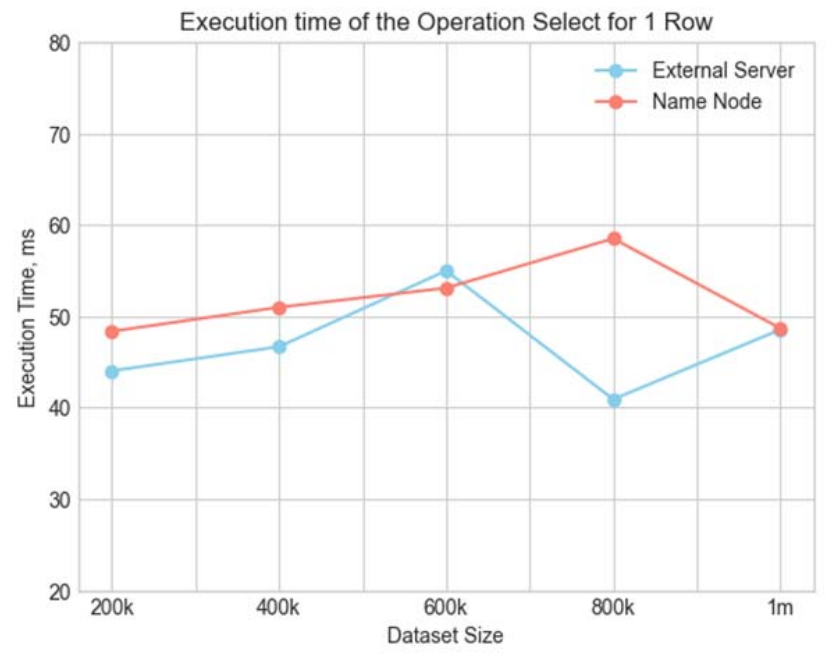

Figure 6. Comparison of select operation for one row (SELECT.1 and SELECT.2)

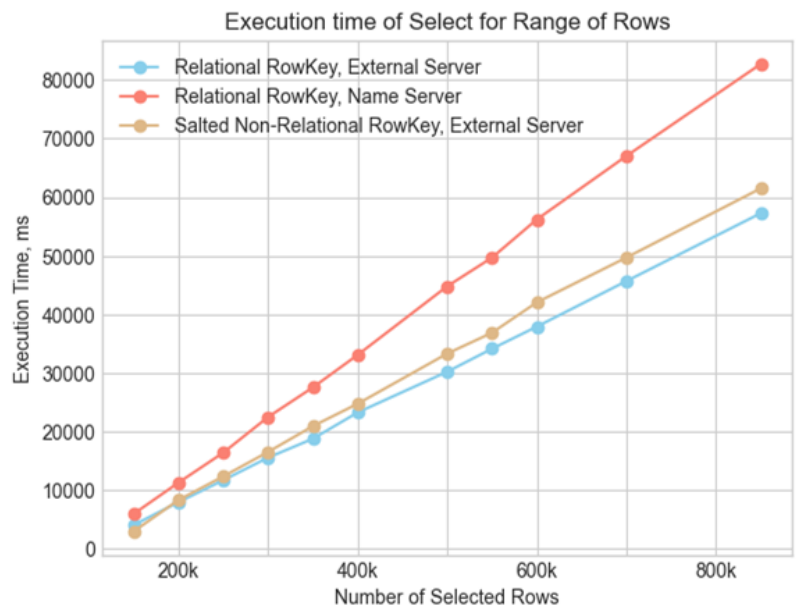

Figure 7. Comparison of select queries with different volume of selected data (SELECT.3, SELECT.3.1, SELECT.3.2, SELECT.4, SELECT.4.1 and SELECT.4.2) 


\section{References}

[1]. Laney, D. (2001). 3D data management: Controlling data volume, velocity and variety. META group research note, $6(70), 1$.

[2]. Lee, I. (2017). Big data: Dimensions, evolution, impacts, and challenges. Business horizons, 60(3), 293-303.

[3]. A Banu, M Yakub (2020), Evolution Of Big Data And Tools For Big Data Analytics, Journal of Interdisciplinary Cycle Research, 12(10), 309-316.

[4]. Tee, J. (2013). Handling the four'V's of big data: volume, velocity, variety, and veracity. TheServerSide. com.

[5]. Embley, D. W., \& Liddle, S. W. (2013, November). Big data-conceptual modeling to the rescue. In International Conference on Conceptual Modeling (pp. 1-8). Springer, Berlin, Heidelberg.

[6]. Das, N., Paul, S., Sarkar, B. B., \& Chakrabarti, S. (2019). NoSQL overview and performance testing of HBase over multiple nodes with MYSQL. In Emerging technologies in data mining and information security (pp. 269-279). Springer, Singapore.
[7]. Hassan, M. U., Yaqoob, I., Zulfiqar, S., \& Hameed, I. A. (2021). A Comprehensive Study of HBase Storage Architecture- A Systematic Literature Review. Symmetry, 13(1), 109.

[8]. George, S. (2013). NoSQL-NOT ONLY SQL. International Journal of Enterprise Computing and Business Systems, 2(2).

[9]. Nitnaware, C., \& Khan, A.(2015). Comparative Analysis of HBase Data Storage Model and RDBMS for Location based Application, International Journal of Science and Research (IJSR), 4(4), 1328-1330.

[10]. Zdravevski, E., Lameski, P., \& Kulakov, A. (2016, February). Row key designs of NoSQL database tables and their impact on write performance. In 2016 24th Euromicro International Conference on Parallel, Distributed, and Network-Based Processing (PDP) (pp. 10-17). IEEE.

[11]. George, L. (2011). HBase: the definitive guide: random access to your planet-size data. " O'Reilly Media, Inc.". 